Gut and Liver, Vol. 6, No. 4, October 2012, pp. 464-470

\title{
Safety and Efficacy of Deep Sedation with Propofol Alone or Combined with Midazolam Administrated by Nonanesthesiologist for Gastric Endoscopic Submucosal Dissection
}

\author{
Seung Yeon Chun, Kyoung Oh Kim, Dong Seon Park, Seong Yeol Kim, Ji Won Park, II Hyun Baek, Jong Hyeok Kim, and \\ Choong Kee Park
}

Department of Internal Medicine, Hallym University College of Medicine, Anyang, Korea

Background/Aims: Endoscopic submucosal dissection (ESD) is accepted as a treatment for gastric neoplasms and usually requires deep sedation. The aim of this study was to evaluate the safety and efficacy profiles of deep sedation induced by continuous propofol infusion with or without midazolam during ESD. Methods: A total of 135 patients scheduled for ESDs between December 2008 and June 2010 were included in this prospective study and were randomly assigned to one of two groups: the propofol group or the combination group (propofol plus midazolam). Results: The propofol group reported only one case of severe hypoxemia with no need of mask ventilation or intubation. Additionally, 18 cases of mild hypotension were observed in the propofol group, and 11 cases were observed in the combination group. The combination group had a lower mean total propofol dose (378 mg vs $466 \mathrm{mg}, \mathrm{p}<0.012$ ), a longer mean recovery time (10.5 minutes vs 7.9 minutes, $p=0.027$ ), and a lower frequency of overall adverse events (32.8\% vs $17.6 \%$, $p=0.042$ ). Conclusions: Deep sedation induced by continuous propofol infusion was shown to be safe during ESD. The combination of continuous propofol infusion and intermittent midazolam injection can decrease the total dose and infusion rate of propofol and the overall occurrence of adverse events. (Gut Liver 2012;6:464-470)

Key Words: Deep sedation; Propofol; Midazolam; Endoscopy; Gastrointestinal

\section{INTRODUCTION}

The number of gastrointestinal endoscopic procedures has in- creased substantially over the last decade. Survey data from the United States suggest that more than 98\% of gastroscopies and colonoscopies were performed with sedation. ${ }^{1}$ In recent years, more complex therapeutic endoscopic procedures have been developed. Endoscopic submucosal dissection (ESD), one of the most difficult endoscopic procedures, is a new and minimally invasive method of treating early gastrointestinal epithelial neoplasms. ${ }^{2}$ A lengthy and potentially uncomfortable endoscopic procedure like ESD usually requires moderate to deep sedation using a method to maintain a stable sedation level. ${ }^{3}$ Deep sedation during ESD with careful monitoring minimizes patient movement, allowing the endoscope to be manipulated precisely with little interruption. ${ }^{4}$

Several sedation protocols have been proposed to improve the acceptability and tolerability of ESD; the propofol sedation protocol is one example. Propofol is a short-acting sedative with a rapid recovery profile. The use of propofol has additional advantages, including the relative ease of safely maintaining a proper sedation level and a good amnesic effect. ${ }^{3}$ These advantages have resulted in increased use of propofol for endoscopic procedures around the world. However, there is an increased risk of cardiopulmonary complications due to the narrow therapeutic range of the drug. ${ }^{4}$ Elderly patients are often candidates for ESD and have a higher risk of complications associated with sedation.

Recently, a number of studies for propofol sedation during ESD by nonanesthesiologist have proven that propofol sedation has similar safety profile compared to conventional sedation with midazolam. ${ }^{3,5,6}$ In these studies, propofol was administrated in bolus or by continuous infusion, and target sedation level was mostly moderate sedation. However, intermittent bolus

Correspondence to: Kyoung Oh Kim

Department of Gastroenterology and Hepatology, Hallym University Sacred Heart Hospital, Hallym University College of Medicine, 22 Gwanpyeong-ro 170beon-gil, Dongan-gu, Anyang 431-796, Korea

Tel: +82-31-380-3710, Fax: +82-31-386-2269, E-mail: kkoimge@hallym.or.kr

Received on May 3, 2012. Accepted on June 22, 2012.

pISSN 1976-2283 eISSN 2005-1212 http://dx.doi.org/10.5009/gnl.2012.6.4.464

@) This is an Open Access article distributed under the terms of the Creative Commons Attribution Non-Commercial License (http://creativecommons.org/licenses/by-nc/3.0) which permits unrestricted non-commercial use, distribution, and reproduction in any medium, provided the original work is properly cited. 
injection of propofol may be theoretically associated with fluctuation of plasma concentration. This variation of plasma level could lead to more frequent complications or delay of procedure due to unsteady sedation level. We hypothesized that continuous infusion of propofol may be associated with a more consistent level of plasma concentration so that maintaining a stable deep sedation level during ESD could be more easily and safely achieved. This study was designed to assess the efficacy and safety profile of deep sedation by continuous propofol infusion in patients underwent ESD, and also aimed to determine the additional advantage of propofol continuous infusion in combination with midazolam.

\section{MATERIALS AND METHODS}

\section{Patients and study design}

This prospective, randomized trial included 135 patients who were scheduled for ESD to remove gastric neoplasms between December 2008 and June 2010 at the Hallym University Sacred Heart Hospital. Adult patients who were 20 to 80 years of age, were classified by the American Society of Anesthesiologists $(\mathrm{ASA})^{7}$ as categories I to III, had gastric adenoma or early gastric cancer (EGC) that had been documented on a previous biopsy, and had a gastric submucosal tumor (SMT) were included. Patients were excluded if they were less than 20 years of age or more than 80 years of age; had an ASA classification of IV to V; had received antiplatelet agents or anticoagulation therapy; or had a history of complications during sedative endoscopy, adverse reactions to propofol and/or midazolam injection, severe obstructive sleep apnea, or allergy to eggs or soybeans.

Using computer-generated random sequencing, all study subjects were randomly assigned to one of two groups: the propofol continuous infusion group (propofol group) or the propofol continuous infusion plus bolus midazolam group (combination group). Verbal and written informed consent was obtained for ESD and sedation. The study was conducted according to the Declaration of Helsinki and was approved by the Institutional Review Board of Hallym University Sacred Heart Hospital. At least one physician with advanced training in basic and cardiac life support was present during each ESD procedure.

\section{Sedation and monitoring protocols}

The patients in the propofol group received a continuous 0.3 $\mathrm{mg} / \mathrm{kg} / \mathrm{min}$ intravenous propofol infusion. The infusion rate was titrated according to the level of sedation. The patients in the combination group received an initial bolus of $2 \mathrm{mg}$ of midazolam and a continuous infusion of $0.3 \mathrm{mg} / \mathrm{kg} / \mathrm{min}$ of intravenous propofol. Trained registered nurses administered all medications under the supervision of the physician performing the endoscopy. The level of sedation was defined according to the ASA classifications: minimal, moderate, and deep sedation. ${ }^{7}$ Minimal sedation is the state in which the patient can respond normally to verbal stimulation. Under moderate sedation, the patient can respond purposefully to verbal commands, either alone or accompanied by light tactile stimulation. Under deep sedation, the patient cannot be aroused easily but responds purposefully following repeated or painful stimulation. In this study, the target level of sedation was deep sedation. Resuscitation equipment was available at all times within the endoscopy unit, and an oncall anesthesiologist was in the building during the procedure.

The patients received supplemental oxygen $(2 \mathrm{~L} / \mathrm{min})$ via a nasal cannula in the endoscopy room, and their vital signs and oxygen saturation were continuously monitored every 5 to 10 minutes using a standard three-lead electrocardiogram, pulse oximetry, and an automatic blood pressure cuff. To assess the accurate sedation level during the ESD procedure, an observer who didn't involve the ESD procedure evaluated the sedation level every 5 minutes using the Modified Observer's Assessment of Awareness/Sedation (MOAA/S) scale. ${ }^{8}$ If MOAA/S score showed the responsiveness score over three or a patient became agitated or produced involuntary movements without any stimulation, the sedation level was considered inappropriate (either moderate or minimal sedation), and the propofol infusion rate was titrated.

\section{Outcome measurement and definitions}

Sedation-related adverse events included hypoxemia and hypotension. Mild hypoxemia was defined as a decline in the oxygen saturation to $<90 \%$ for less than 30 seconds that is corrected with supplemental oxygen and a decrease in the propofol infusion rate. Severe hypoxemia was defined as a decline in the oxygen saturation to $<90 \%$ for more than 30 seconds that was not corrected with the aforementioned method. Severe hypoxemia was managed by discontinuing the propofol infusion and interrupting the procedure to secure the airway. If there was no response, bag-mask ventilation and/or endotracheal intubation were considered.

Hypotension was defined as a decline in blood pressure to $<90 / 50 \mathrm{~mm} \mathrm{Hg}$ or a decrease in the baseline blood pressure by $>20 \%$. Mild hypotension was corrected with a saline infusion or a decrease in the propofol infusion rate. Severe hypotension was defined as hypotension that could not be corrected with the aforementioned method and lasted for more than 5 minutes. It was managed by discontinuing the propofol infusion, administering large volumes of intravenous fluid, and using the Trendelenburg position.

The procedure time was the time from the entry of the sedatives to the withdrawal of endoscope following submucosal dissection. The recovery state was defined as a condition in which the patient can respond to verbal orders. The recovery time was the time from the cessation of the drug infusion to the recovery state. After the procedure, the degree of satisfaction of both the endoscopist and the patient was evaluated using an endoscopist score and a patient score, respectively. The endoscopist score 
Table 1. Baseline Characteristics of the Patients

\begin{tabular}{lcccc}
\hline \multicolumn{1}{c}{ Characteristic } & $\begin{array}{c}\text { Total } \\
(\mathrm{n}=135)\end{array}$ & $\begin{array}{c}\text { Propofol } \\
(\mathrm{n}=67)\end{array}$ & $\begin{array}{c}\text { Propofol+ } \\
\text { Midazolam } \\
(\mathrm{n}=68)\end{array}$ & $\begin{array}{c}\mathrm{p}- \\
\text { value }\end{array}$ \\
\hline Age, yr & $63 \pm 10.57$ & $64 \pm 9.27$ & $63 \pm 11.72$ & 0.418 \\
Gender, M:F & $94: 41$ & $45: 22$ & $49: 19$ & 0.498 \\
Weight, kg & $62.5 \pm 11.09$ & $61.7 \pm 9.26$ & $63.4 \pm 12.63$ & 0.382 \\
Alcohol, mg/wk & $82 \pm 193.81$ & $59 \pm 175.68$ & $105 \pm 208.84$ & 0.182 \\
Systolic BP & $131 \pm 18.03$ & $132 \pm 17.32$ & $129 \pm 18.67$ & 0.267 \\
Diastolic BP & $74 \pm 12.72$ & $75 \pm 14.25$ & $73 \pm 11.08$ & 0.447 \\
Underlying diseases & & & & 0.852 \\
Cardiac disease & 10 & 4 & 6 & \\
Pulmonary disease & 4 & 1 & 3 & \\
Hypertension & 46 & 22 & 24 & \\
Diabetes mellitus & 9 & 5 & 4 & \\
Other & 9 & 5 & 4 & \\
\hline
\end{tabular}

Data are presented as number or mean \pm SD.

$\mathrm{M}$, male; F, female; BP, blood pressure.

was a scale from one (unsatisfactory) to four points (very satisfactory). The patient score was a scale from zero (very severe discomfort) to 10 points (no discomfort).

\section{Statistical analyses}

The $\chi^{2}$ test or Fisher's exact test was used for comparisons of categorical data, and the t-test was used for continuous data. A p-value of $<0.05$ was considered to be statistically significant. All data were analyzed using the SPSS version 16.0 for Windows (SPSS Korea, Seoul, Korea).

\section{RESULTS}

A total of 135 patients were enrolled in this prospective study; 67 patients were randomly assigned to the propofol group, and 68 were assigned to the combination group. The patient characteristics of both groups are shown in Table 1 . There were no significant differences between the two groups in terms of age, gender, body weight, baseline blood pressure, underlying diseases (diabetes mellitus, hypertension, cardiovascular disease, or pulmonary disease), or ASA score. The combination group was more likely to consume higher volumes of alcohol than the propofol group, but there was no significant difference between the groups.

The characteristics of the gastric lesions are shown in Table 2. The diagnostic pathology results were adenoma, EGC, or SMT. In the propofol group, there were 44 adenomas, 17 EGCs, and six SMTs. In the combination group, there were 39 adenomas, 23 EGCs, and six SMTs. The lesion was more commonly located in the lower third of the stomach in both groups. The average specimen size was $34.74 \pm 13.65 \mathrm{~mm}$ in the propofol group and $42.74 \pm 16.86 \mathrm{~mm}$ in the combination group $(\mathrm{p}=0.003)$.
Table 2. Characteristics of the Gastric Lesions

\begin{tabular}{lcccc}
\hline \multicolumn{1}{c}{ Characteristic } & Total & Propofol & $\begin{array}{c}\text { Propofol+ } \\
\text { Midazolam }\end{array}$ & $\begin{array}{c}\text { p- } \\
\text { value }\end{array}$ \\
\hline Diagnosis & & & & 0.587 \\
$\quad$ Adenoma & 83 & 44 & 39 & \\
EGC & 40 & 17 & 23 & \\
SMT & 12 & 6 & 6 & \\
Location & & & & 0.131 \\
Upper & 15 & 11 & 4 & \\
Mid & 39 & 19 & 20 & \\
Lower & 81 & 37 & 44 & \\
Specimen size, $m$ mm & $38.90 \pm 15.82$ & $34.74 \pm 13.65$ & $42.74 \pm 16.86$ & 0.003 \\
\hline
\end{tabular}

Data are presented as number or mean \pm SD.

EGC, early gastric cancer; SMT, submucosal tumor.

Table 3. The Efficacy Profile of Propofol Group versus Combination (Propofol and Midazolam) Group

\begin{tabular}{|c|c|c|c|c|}
\hline & Total & Propofol & $\begin{array}{l}\text { Propofol+ } \\
\text { Midazolam }\end{array}$ & $\begin{array}{c}\mathrm{p}- \\
\text { value }\end{array}$ \\
\hline Procedure time, $\min$ & $55 \pm 34.49$ & $57 \pm 34.34$ & $53 \pm 34.79$ & 0.528 \\
\hline Total dose, mg & $422 \pm 215.36$ & $466 \pm 200.55$ & $378 \pm 220.66$ & 0.012 \\
\hline $\begin{array}{c}\text { Infusion rate, } \\
\mathrm{mg} / \mathrm{kg} / \mathrm{min}\end{array}$ & $0.139 \pm 0.05$ & $0.160 \pm 0.06$ & $0.118 \pm 0.03$ & $<0.01$ \\
\hline Pt. satisfaction score & $10 \pm 0.90$ & $9 \pm 0.96$ & $10 \pm 0.84$ & 0.704 \\
\hline $\begin{array}{l}\text { Dr. satisfaction } \\
\text { score }\end{array}$ & $4 \pm 0.67$ & $4 \pm 0.78$ & $4 \pm 0.54$ & 0.652 \\
\hline Recovery time, min & $9.2 \pm 6.87$ & $7.9 \pm 3.78$ & $10.5 \pm 8.74$ & 0.027 \\
\hline
\end{tabular}

Data are presented as mean \pm SD.

Pt., patient; Dr., doctor (endoscopist).

Table 3 shows that the mean procedure time was not significantly different between the two groups ( 57 minutes vs 53 minutes, $\mathrm{p}=0.528$ ). The mean total dose of propofol administered was lower in the combination group than in the propofol group (378 $\pm 220 \mathrm{mg}$ vs $466 \pm 200 \mathrm{mg}, \mathrm{p}=0.012$ ). The mean propofol infusion rate was lower in the combination group $(0.118 \pm 0.03$ $\mathrm{mg} / \mathrm{kg} / \mathrm{min}$ vs $0.160 \pm 0.06 \mathrm{mg} / \mathrm{kg} / \mathrm{min}, \mathrm{p}<0.001)$, but the combination group had a longer mean recovery time $(7.9 \pm 3.78$ minutes vs $10.5 \pm 8.74$ minutes, $\mathrm{p}=0.027$ ) (Fig. 1). Based on a 10-point visual analogue scale, the patients in both groups showed similar degrees of overall satisfaction.

The adverse events are shown in Table 4. There were three cases of mild hypoxemia in the propofol group and one in the combination group $(p=0.362)$, and there was one case of severe hypoxemia in the propofol group. Mild hypotension was observed in 18 cases in the propofol group and 11 cases in the combination group $(\mathrm{p}=0.144)$. There was no severe hypotension in either group, and no patients required mask ventilation with an Ambu bag or intubation. Frequency of each adverse event was similar between two groups. But total adverse event rate of 

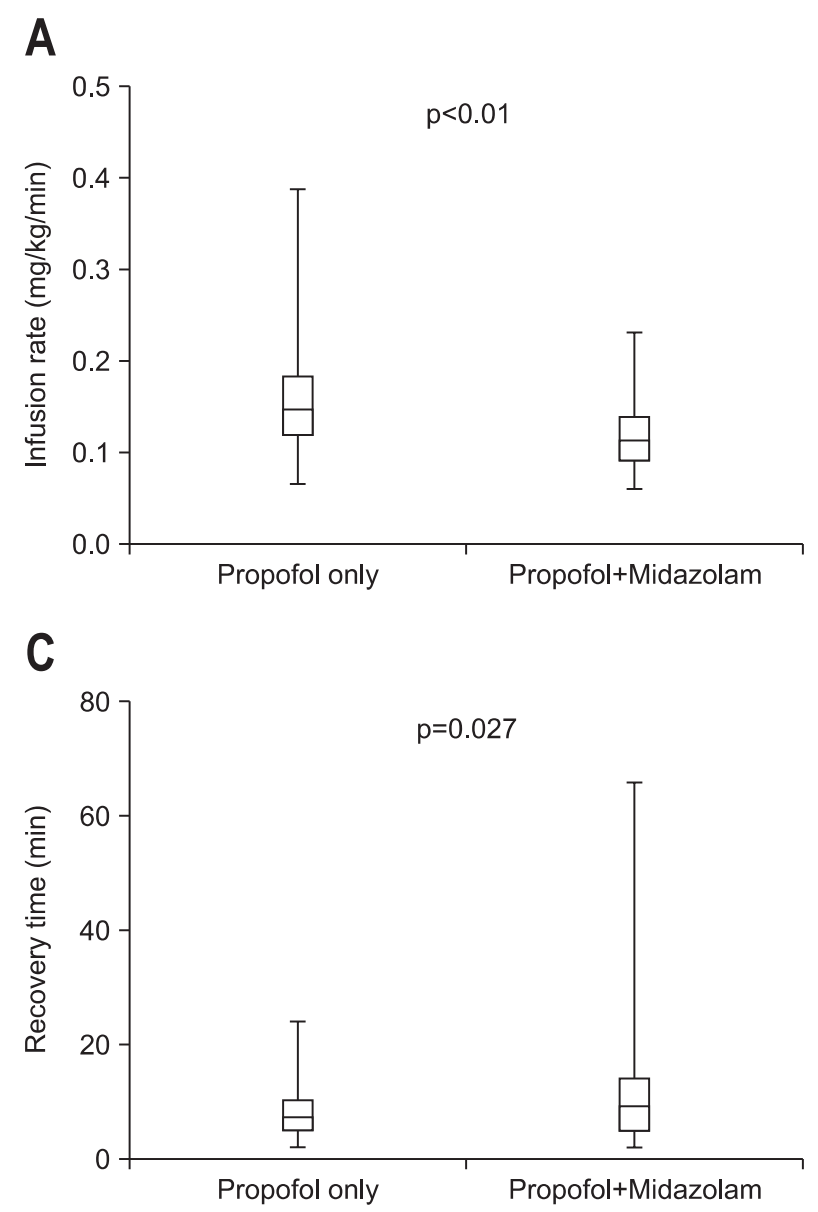

Table 4. Adverse Events

\begin{tabular}{lcccc}
\hline & Total & Propofol & $\begin{array}{c}\text { Propofol+ } \\
\text { Midazolam }\end{array}$ & $\begin{array}{c}\mathrm{p}- \\
\text { value }\end{array}$ \\
\hline Hypoxemia & $5(3.7)$ & $4(6.0)$ & $1(1.5)$ & 0.166 \\
$\quad$ Mild & $4(3)$ & $3(4.5)$ & $1(1.5)$ & \\
$\quad$ Severe & $1(0.7)$ & $1(1.5)$ & $0(0)$ & \\
Hypotension & $29(21.5)$ & $18(26.9)$ & $11(16.2)$ & 0.131 \\
$\quad$ Mild & $29(21.5)$ & $18(26.9)$ & $11(16.2)$ & \\
$\quad$ Severe & $0(0)$ & $0(0)$ & $0(0)$ & \\
Total adverse events & $34(25.2)$ & $22(32.8)$ & $12(17.6)$ & 0.042 \\
\hline
\end{tabular}

Data are presented as number $(\%)$.

combination group was significantly less than that of propofol alone group (32.8\% vs $17.6 \%, \mathrm{p}=0.042$ ).

Table 5 compares the study participants who had an adverse event (adverse event group) with those who did not (noadverse-event group). The mean total propofol dose was $394 \mathrm{mg}$ in the adverse event group and $431 \mathrm{mg}$ in the no-adverse-event group. The propofol infusion rate was $0.136 \mathrm{mg} / \mathrm{kg} / \mathrm{min}$ in the adverse event group and $0.140 \mathrm{mg} / \mathrm{kg} / \mathrm{min}$ in the no-adverseevent group. The study results indicate that there was no correlation between the propofol dose or infusion rate with adverse events. The total procedure time was longer in the adverse event

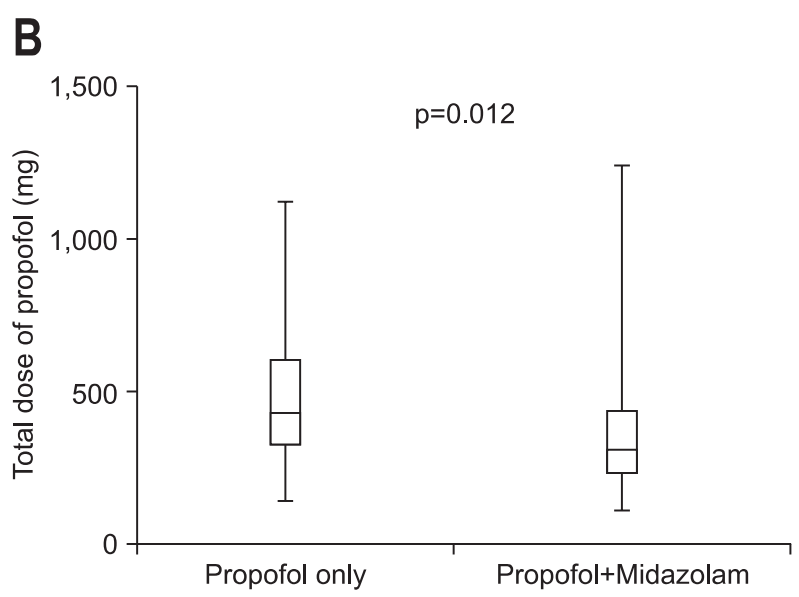

Fig. 1. Comparisons of the efficacy profile between the propofol group and the combination group. (A) Total dose of propofol administered $(p=0.012)$. (B) Propofol infusion rate $(p<0.01)$. (C) Recovery time of the patients $(p=0.027)$.

Table 5. Adverse Event Group versus the Non-Adverse Event Group

\begin{tabular}{lccc}
\hline & $\begin{array}{c}\text { Adverse event } \\
(\mathrm{n}=34)\end{array}$ & $\begin{array}{c}\text { Non-adverse } \\
\text { event } \\
(\mathrm{n}=101)\end{array}$ & $\mathrm{p}$-value \\
\hline Age, yr & $68 \pm 10.481$ & $62 \pm 10.324$ & 0.012 \\
Gender, M:F & $25: 9$ & $69: 32$ & 0.829 \\
Body weight, Kg & $60.6 \pm 11.017$ & $63.1 \pm 11.104$ & 0.289 \\
ASA score, I:II:III & $11: 19: 4$ & $50: 47: 4$ & 0.032 \\
Alcohol, mg/wk & $89 \pm 237.651$ & $80 \pm 179.804$ & 0.757 \\
Total propofol dose, mg & $394 \pm 225.110$ & $431 \pm 213.053$ & 0.533 \\
Propofol infusion rate, & $0.136 \pm 0.049$ & $0.140 \pm 0.054$ & 0.322 \\
$\quad$ mg/kg/min & & & \\
Procedure time, min & $59 \pm 45.432$ & $54 \pm 30.602$ & 0.414 \\
Underlying disease $(+)$ & 20 & 55 & 0.717 \\
\hline
\end{tabular}

Data are presented as mean \pm SD or number.

M, male; F, female; ASA, American Society of Anesthesiologists.

group than in the no-adverse-event group, but did not reach significance ( 59 minutes vs 54 minutes, $\mathrm{p}=0.414$ ). The mean age was higher in the adverse event group $(68 \pm 10.48$ years vs $62 \pm 10.32$ years, $p=0.012$ ), and cases with an ASA class of more than II were more frequent in the adverse event group $(\mathrm{p}=0.032)$. 


\section{DISCUSSION}

Our results show that propofol was successfully administrated by continuous infusion for induction and maintenance of deep sedation during ESD. The use of propofol for endoscopic sedation by nonanesthesiologist providers has increased markedly during the last decade, ${ }^{1}$ and recent survey data suggest that propofol sedation is associated with a lower risk of complications and serious adverse events during standard endoscopy compared to conventional sedation. The safety and efficacy of propofol for other complex therapeutic endoscopic procedures, such as endoscopic retrograde cholangiopancreatography and endoscopic ultrasound, has already been established by several studies. ${ }^{9-11}$ Nevertheless, the most effective and safest sedative agent and sedation protocol for ESD has not yet been clearly established.

Propofol is a potent sedative agent with a narrow therapeutic window. It has a rapid onset of action and short recovery time. Propofol also provided significantly better patient cooperation than midazolam. ${ }^{12,13}$ However, this agent occasionally decreases systemic vascular resistance, cardiac contractility, and ultimately cardiac output without a concomitant change in heart rate, and respiratory depression can also occur., ${ }^{5,14,15}$

Recently, several studies have revealed most adverse events associated with propofol sedation were mild and often transient during advanced interventional endoscopic procedure. ${ }^{12,16-18} \mathrm{~A}$ randomized study in Japan, which compared continuous propofol infusion with intermittent midazolam injection during ESD for EGC, found that propofol is a safe and effective sedative agent and that patients treated with propofol had a quicker recovery than those who were treated with midazolam. ${ }^{3}$ In those studies, however, sedation was mostly targeted to a moderate level and did not monitor an accurate sedation level during the procedure. Moderate sedation with propofol, especially in bolus injection, may frequently move into lighter or deeper sedation due to inconstant plasma concentration of propofol, disturbing the ESD procedure. In our studies, an assessment of an accurate sedation level was performed using a MOAA/S scale every 5 minutes during ESD procedure. Similar to other studies for propofol sedation targeting a moderate sedation, our results showed that deep sedation with continuous propofol infusion during ESD had a relatively low incidence of cardiopulmonary complications. There was only one case of severe hypoxemia, and that case did not require mask ventilation or intubation. Both patients and endoscopists gave high satisfaction scores after ESD, and there was no interruption or delay of the procedure.

Propofol may be administered alone, but in clinical practice, it is commonly administered in combination with low doses of midazolam or a narcotic. ${ }^{19}$ Because of the synergic effects of propofol, its dose can be reduced significantly when it is used in combination with midazolam and narcotics, possibly improving its safety. ${ }^{20,21}$ Recent studies have assessed the safety and effi- cacy of sedation with co-administration of propofol and opiates or midazolam to maintain a moderate sedation level. Similar to the results for propofol alone, the pooled analysis showed that the combination protocol resulted in a low incidence of hypoxemia (10\%) but a relatively high incidence of nausea (26\%) and memory of the procedure (66\%). ${ }^{22}$

Our study demonstrated that the deep sedation using combination protocol for ESD was as safe and effective as sedation by continuous infusion of propofol alone. Furthermore, sedation could be maintained with a significantly lower dose of propofol in the combination group. A significantly slower mean infusion rate in the combination group was also observed. Because propofol is a potent respiratory depressant, a significant reduction in the amount of propofol required for deep sedation could theoretically decrease the risk of respiratory depression. In our study, each of hypotension and hypoxemia showed no difference between two groups, but total adverse event rate of the combination group was significantly lower than propofol infusion alone group. Low doses and slow infusion rate of propofol may lead to a low incidence of adverse events. However, the combination protocol had a longer recovery time even though we only administered midazolam as a bolus at the start of the procedure to minimize the delay in recovery. A recent study showed that midazolam has a pharmacodynamic interaction during prolonged propofol infusion. ${ }^{23}$ In this study, the results indicated that midazolam, at sedative levels, increases blood propofol concentrations by $25 \%$ and that propofol reduces the distribution and clearance of midazolam in a concentration-dependent manner. This synergetic effect of these two drugs could explain a prolonged recovery time in the combination group.

Additionally, one point of clinical importance in our study is that trained nurses administered the propofol under the supervision of an experienced endoscopist. Recent large-scale trials of nurse-administered propofol sedation (NAPS) have suggested that appropriately trained nurses could assist the endoscopist in propofol administration and sedation monitoring, clearly saving resources. ${ }^{24-27}$ Our study showed that the cardiopulmonary complication rate from sedation was $25.2 \%$ and that all of the complications were transient and clinically insignificant. However, NAPS is still a subject of debate, and we cannot completely exclude the potential risk of cardiopulmonary complications of NAPS during ESD, even though we did not observe any major complications. Previous studies have suggested that NAPS is a safe method of sedation, at least in a tertiary hospital gastroenterology department, provided that training and the use of emergency measures to counteract its adverse effects is adequate. $^{25}$

There are several limitations in our study. First, older and high-risk patients (ASA category IV) were excluded, and the proportion of ASA category III patients was small. This could be a cause of the relatively low incidence of cardiopulmonary complications. Second, we could not apply a double blind design; 
the endoscopist could not be blinded, which might have biased the satisfaction assessment. Third, the sample size was too small to exclude rare adverse events or to adequately power the study to demonstrate the safety and efficacy of deep sedation by propofol. Finally, we did not directly compare the continuous propofol infusion and intermittent bolus injection of propofol, so further studies to compare the continuous infusion and intermittent bolus injection of propofol are needed to investigate the most effective and safe sedation protocol for ESD.

The aim of this study was to evaluate and compare the safety and efficacy of deep sedation induced by the continuous infusion of propofol administrated by a nonanesthesiologist during ESD. Using an adequate monitoring system, safe and efficient endoscopic procedures were performed with no serious adverse events using propofol. The combination protocol of continuous propofol infusion and intermittent intravenous midazolam injection can decrease the adverse events. However, further studies are needed on the specific adverse effects of propofol or other sedative agents used for deep sedation during complicated endoscopic procedures.

\section{CONFLICTS OF INTEREST}

No potential conflict of interest relevant to this article was reported.

\section{REFERENCES}

1. Cohen LB, Wecsler JS, Gaetano JN, et al. Endoscopic sedation in the United States: results from a nationwide survey. Am J Gastroenterol 2006;101:967-974.

2. Imagawa A, Okada H, Kawahara $\mathrm{Y}$, et al. Endoscopic submucosal dissection for early gastric cancer: results and degrees of technical difficulty as well as success. Endoscopy 2006;38:987-990.

3. Kiriyama S, Gotoda T, Sano H, et al. Safe and effective sedation in endoscopic submucosal dissection for early gastric cancer: a randomized comparison between propofol continuous infusion and intermittent midazolam injection. J Gastroenterol 2010;45:831837.

4. Skues MA, Prys-Roberts C. The pharmacology of propofol. J Clin Anesth 1989;1:387-400.

5. Yamagata T, Hirasawa D, Fujita N, et al. Efficacy of propofol sedation for endoscopic submucosal dissection (ESD): assessment with prospective data collection. Intern Med 2011;50:1455-1460.

6. Cho YS, Seo E, Han JH, et al. Comparison of midazolam alone versus midazolam plus propofol during endoscopic submucosal dissection. Clin Endosc 2011;44:22-26.

7. American Society of Anesthesiologists Task Force on Sedation and Analgesia by Non-Anesthesiologists. Practice guidelines for sedation and analgesia by non-anesthesiologists. Anesthesiology 2002;96:1004-1017.

8. Paspatis GA, Chainaki I, Manolaraki MM, et al. Efficacy of bispec- tral index monitoring as an adjunct to propofol deep sedation for ERCP: a randomized controlled trial. Endoscopy 2009;41:10461051.

9. Jung M, Hofmann C, Kiesslich R, Brackertz A. Improved sedation in diagnostic and therapeutic ERCP: propofol is an alternative to midazolam. Endoscopy 2000;32:233-238.

10. Wehrmann T, Kokabpick S, Lembcke B, Caspary WF, Seifert H. Efficacy and safety of intravenous propofol sedation during routine ERCP: a prospective, controlled study. Gastrointest Endosc 1999;49:677-683.

11. Yusoff IF, Raymond G, Sahai AV. Endoscopist administered propofol for upper-GI EUS is safe and effective: a prospective study in 500 patients. Gastrointest Endosc 2004;60:356-360.

12. Horn E, Nesbit SA. Pharmacology and pharmacokinetics of sedatives and analgesics. Gastrointest Endosc Clin N Am 2004;14:247268.

13. Kanto J, Gepts E. Pharmacokinetic implications for the clinical use of propofol. Clin Pharmacokinet 1989;17:308-326.

14. Larijani GE, Gratz I, Afshar M, Jacobi AG. Clinical pharmacology of propofol: an intravenous anesthetic agent. DICP 1989;23:743749.

15. Short TG, Plummer JL, Chui PT. Hypnotic and anaesthetic interactions between midazolam, propofol and alfentanil. Br J Anaesth 1992;69:162-167.

16. Qadeer MA, Vargo JJ, Khandwala F, Lopez R, Zuccaro G. Propofol versus traditional sedative agents for gastrointestinal endoscopy: a meta-analysis. Clin Gastroenterol Hepatol 2005;3:1049-1056.

17. Carlsson U, Grattidge P. Sedation for upper gastrointestinal endoscopy: a comparative study of propofol and midazolam. Endoscopy 1995;27:240-243.

18. Sipe BW, Rex DK, Latinovich D, et al. Propofol versus midazolam/ meperidine for outpatient colonoscopy: administration by nurses supervised by endoscopists. Gastrointest Endosc 2002;55:815-825.

19. Cohen LB, Hightower CD, Wood DA, Miller KM, Aisenberg J. Moderate level sedation during endoscopy: a prospective study using low-dose propofol, meperidine/fentanyl, and midazolam. Gastrointest Endosc 2004;59:795-803.

20. Rex DK. Review article: moderate sedation for endoscopy: sedation regimens for non-anaesthesiologists. Aliment Pharmacol Ther 2006;24:163-171.

21. Cohen LB, Dubovsky AN, Aisenberg J, Miller KM. Propofol for endoscopic sedation: a protocol for safe and effective administration by the gastroenterologist. Gastrointest Endosc 2003;58:725-732.

22. McQuaid KR, Laine L. A systematic review and meta-analysis of randomized, controlled trials of moderate sedation for routine endoscopic procedures. Gastrointest Endosc 2008;67:910-923.

23. Lichtenbelt BJ, Olofsen E, Dahan A, van Kleef JW, Struys MM, Vuyk J. Propofol reduces the distribution and clearance of midazolam. Anesth Analg 2010;110:1597-1606.

24. Rex DK, Overley C, Kinser K, et al. Safety of propofol administered by registered nurses with gastroenterologist supervision in 2000 endoscopic cases. Am J Gastroenterol 2002;97:1159-1163. 
25. Heuss LT, Schnieper P, Drewe J, Pflimlin E, Beglinger C. Risk stratification and safe administration of propofol by registered nurses supervised by the gastroenterologist: a prospective observational study of more than 2000 cases. Gastrointest Endosc 2003;57:664671.

26. Heuss LT, Drewe J, Schnieper P, Tapparelli CB, Pflimlin E, Beg- linger C. Patient-controlled versus nurse-administered sedation with propofol during colonoscopy: a prospective randomized trial. Am J Gastroenterol 2004;99:511-518.

27. Rex DK, Heuss LT, Walker JA, Qi R. Trained registered nurses/ endoscopy teams can administer propofol safely for endoscopy. Gastroenterology 2005;129:1384-1391. 